\section{Migrating Surgical Suture Material in the Duodenum After Cholecystectomy}

This report presents a rare pathology of the migration of surgical suture material applied during biliary surgery to the duodenal bulb. The first case was a 21 -year-old woman who had previously undergone cholecystectomy and choledochotomy, and was readmitted later due to epigastric pain. ERCP was negative. Panendoscopy revealed a yellowish lesion in the duodenal bulb, with inflammatory surroundings. After the extraction of the material, the specimen proved to be interrupted surgical sutures.

A 47-year-old woman also underwent cholecystectomy. Nine months later she experienced epigastric pain. Upper panendoscopy showed yellowish material in the bulb of the duodenum, surrounded by an ulcer (Figure 1). The extracted specimen proved to be an inflammatory suture granuloma. Both patients became free of symptoms after the extraction of the suture material, and follow-up endoscopy was normal.

The third case was a 47-year-old woman who underwent cholecystectomy and two years later suffered pain in the right subcostal region. Ultrasonography revealed choledocholithiasis. At endoscopy, surgical suture material was observed with normal surroundings in the duodenal bulb (Figure 2). The attempt to remove the suture material was unsuccessful. Endoscopic sphincterotomy was performed, and clearance of the common bile duct was achieved. The patient did not attend for follow-up. Among 34,886 upper panendoscopies, we observed three cases of suture migration to the duodenal bulb following cholecystectomy. Reviewing the operative documentation, we found no intestinal injuries that required the use of suture material, so that the migration of the suture material must have occurred by an unknown mechanism.

I. Szántó ${ }^{\text {, E.M. Gamal }}$, A. Bajtai ${ }^{2}$, J. Banai ${ }^{1}$, J. Kiss ${ }^{1}$ ${ }^{1}$ Department of Surgery and ${ }^{2}$ Institute of Pathology Postgraduate Medical School, Budapest, Hungary

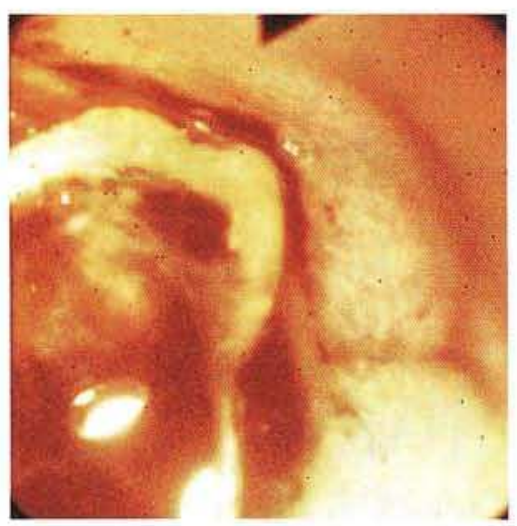

Figure 1:

Suture material surrounded by ulcer.

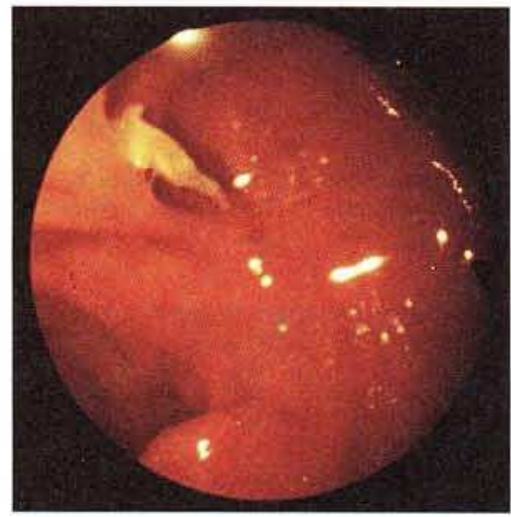

Figure 2:

Surgical suture material in the duodenal bulb.

Corresponding Author

I. Szántó, Department of Surgery, Postgraduate Medical School, Szabolcs u. 35, P.O. Box 112, 1389 Budapest, Hungary 